\title{
Site-Specific Ventricular Tachycardia Inducibility
}

Asad Aboud ${ }^{1}$, Robert Abraham ${ }^{1}$, oluwaseun adeola ${ }^{1}$, Ikutaro Nakajima ${ }^{2}$, Ryohsuke Narui $^{3}$, Tomofumi Nakamura ${ }^{4}$, Arvindh Kanagasundram ${ }^{1}$, Travis Richardson ${ }^{1}$, and William Stevenson $^{5}$

${ }^{1}$ Vanderbilt University Medical Center

${ }^{2}$ Saint Marianna University School of Medicine

${ }^{3}$ The Jikei University School of Medicine

${ }^{4}$ Tokyo Metropolitan Toshima Hospital

${ }^{5}$ Vanderbilt Heart and Vascular Institute

May 10, 2021

\begin{abstract}
Introduction Programmed electrical stimulation is an essential part of VT ablation procedures but VT is not always inducible, usually for reasons that are not clear. We sought to review pacing site-specific failure of programmed electrical stimulation (PES) to induce scar-related ventricular tachycardia (VT). Methods A series of patients in whom aggressive programmed stimulation from traditional RV pacing sites failed to induce VT, but VT was easily inducible from a non-traditional site are reviewed. Computer simulations in a simple 2-dimensional model of reentry were performed. Results Six patients who had no inducible sustained VT from the RV apex/outflow tract with at least 3 extrastimuli, but relatively easily induced VT from the LV, basal $\mathrm{RV}$, epicardium, or atrium are described. In 5 of these patients, the site that induced VT was closer to the likely reentry circuit region based on mapping and ablation. Computer simulations illustrated that the spatial relation between the pacing site and the entrance and exits of a reentry isthmus can determine the ease of initiation of reentry by determining the time available for recovery of excitability at the initial region of block. Conclusions The site of PES has a marked effect on inducibility of VT in some patients such that PES from the RV apex and outflow regions will fail to expose clinically relevant VTs. The frequency with which this occurs is not certain. Stimulation from alternative sites is a reasonable consideration in selected patients.
\end{abstract}

\section{Site-Specific Ventricular Tachycardia Inducibility}

Short title: Site Specific VT Inducibility

\section{Authors Names :}

Asad A. Aboud, MD ${ }^{1}$, Robert L. Abraham, MD ${ }^{1}$, Oluwaseun Adeola, $\mathrm{MD}^{1}$, Ikutaro Nakajima, MD, $\mathrm{PhD}^{2}$; Ryohsuke Narui, MD, $\mathrm{PhD}^{3}$, Tomofumi Nakamura, $\mathrm{MD}^{4}$, Arvindh N. Kanagasundram, $\mathrm{MD}^{1}$, Travis Richardson, $\mathrm{MD}^{1}$, William G. Stevenson, $\mathrm{MD}^{1}$

1. Vanderbilt Heart and Vascular Institute, Nashville, TN, USA

2. Division of Cardiology, St. Marianna University School of Medicine, Kawasaki, Japan.

3. Division of Cardiology, The Jikei University School of Medicine, Tokyo, Japan.

4. Heart Center, Tokyo Metropolitan Toshima Hospital, Tokyo, Japan.

\section{Corresponding author:}

Asad Aboud, MD

Clinical Fellow, Cardiac Electrophysiology 
Vanderbilt University Medical Center

1211 Medical Center Drive MCE 5414

Nashville, TN 37232-8802

Asad.a.al.aboud@vumc.org

\section{Sources of Funding: None}

Disclosure: The authors have no conflicts to disclose.

Abstract

\section{Introduction}

Programmed electrical stimulation is an essential part of VT ablation procedures but VT is not always inducible, usually for reasons that are not clear. We sought to review pacing site-specific failure of programmed electrical stimulation (PES) to induce scar-related ventricular tachycardia (VT).

\section{Methods}

A series of patients in whom aggressive programmed stimulation from traditional RV pacing sites failed to induce VT, but VT was easily inducible from a non-traditional site are reviewed. Computer simulations in a simple 2-dimensional model of reentry were performed.

\section{Results}

Six patients who had no inducible sustained VT from the RV apex/outflow tract with at least 3 extrastimuli, but relatively easily induced VT from the LV, basal RV, epicardium, or atrium are described. In 5 of these patients, the site that induced VT was closer to the likely reentry circuit region based on mapping and ablation. Computer simulations illustrated that the spatial relation between the pacing site and the entrance and exits of a reentry isthmus can determine the ease of initiation of reentry by determining the time available for recovery of excitability at the initial region of block.

\section{Conclusions}

The site of PES has a marked effect on inducibility of VT in some patients such that PES from the RV apex and outflow regions will fail to expose clinically relevant VTs. The frequency with which this occurs is not certain. Stimulation from alternative sites is a reasonable consideration in selected patients.

Keywords: Ventricular Tachycardia, Programmed Electric Stimulation, Site-Specific Induction, VT Ablation, VT Simulation

\section{Introduction}

Programmed electrical stimulation (PES) of the ventricle is an important tool for initiation of ventricular tachycardia (VT) during ablation procedures. It is used to confirm the diagnosis, guide the procedural approach, and assess the effect of ablation. ${ }^{1}$ The absence of inducible VT following VT ablation is associated with lower risk of recurrence. ${ }^{2}$ Some observations, however, call into question the reliability of programmed stimulation for these purposes. Some VTs are not inducible in the electrophysiology laboratory despite aggressive PES. When this occurs, ablation is typically guided by targeting the presumptive VT substrate based on electroanatomic voltage mapping, electrogram characteristics, and/or pace-mapping. Secondly, it is well recognized that a clinical VT may be rendered no longer inducible immediately after ablation, and then be inducible again a few days later. In one study this occurred in $18.2 \%$ of patients for whom ablation was deemed acutely successful. ${ }^{3}$

Inducibility of VT is related to the aggressiveness of the PES. Using multiple drive cycle lengths and up to 3 or 4 extrastimuli, PES has a high sensitivity for inducing VT in patients who have had this arrhythmia spontaneously. ${ }^{4}$ The site of stimulation also plays a role. The initial ventricular stimulation site is typically 
the RV apex (RVA) and then the RV outflow tract (RVOT) if VT is not inducible from the RVA. This approach is widely adopted after earlier studies demonstrated the incremental yield of programmed electric stimulation (PES) from two RV sites. ${ }^{5}$ Before the era of catheter ablation, Josephson and colleagues reported that up to $11 \%$ of patients studied in the electrophysiology laboratory for VT had VTs that could be induced only with left ventricular (LV) and not with right ventricular stimulation. ${ }^{6}$ While their series did not find any electrocardiographic predictors of the need for LV stimulation, it is likely that site-specific inducibility varies with the location of the reentry circuit as well as with tissue conduction properties.

We describe here a case series of patients in whom inducibility was exquisitely sensitive to the site of stimulation and discuss the implications for VT ablation studies and patient management.

Methods

From patients referred for VT ablation, 6 patients were identified who had VT inducible from outside of the right ventricular apical or outflow region. Patients underwent electrophysiological studies and ablation according to the previously published method. ${ }^{7-10}$ An informed consent was obtained prior to the ablation procedure. Either Monitored Anesthesia Care (MAC) or general anesthesia was delivered by a dedicated anesthesia team throughout the procedure. Electroanatomic maps were constructed using a 3D mapping system (CARTO3, Biosense Webster) and intracardiac echocardiography (ICE). Programmed electric stimulation protocol involved 1-3 extrastimuli after two paced cycle length (typically 400 and $600 \mathrm{~ms}$ ) from two sites (typically the RV apex or septum and the RVOT). Additional stimulation sites and use of 4 extrastimuli were chosen at the discretion of the operator. Data collection was performed under protocols approved by the institutional review board. This research adheres to the ethical guidelines specified in Helsinki Declaration as revised in 2013.

\section{Computer simulation}

A simple, anatomically fixed reentry circuit was constructed in a custom software model of a cardiac tissue plane using the Matlab programming environment (Matlab R2020a, The Mathworks, Inc., Natick, MA). A propagation model was created consisting of an $1281 \times 1281$ matrix of excitable elements. The nominal dimensions of the simulation matrix are $40 \mathrm{~mm}$ x $40 \mathrm{~mm}$ with an imbedded $20 \mathrm{~mm}$ diameter circular scar. Within the non-conducting scar is an asymmetric gap of conducting elements. The matrix is isotropic except for a difference in refractory period within the scar gap. The excitable elements are $31.25 \mu \mathrm{m}$ in diameter, though this is an arbitrary size chosen to provide as dense a matrix as possible within the constraints of the available computational resources. To allow more physiologic circular wavefronts within the rectangular simulation matrix, each activated element activates a roughly circular cluster of adjacent elements. The 16element-radius size of this cluster was chosen to allow a wavefront as smooth as possible within the available computational constraints. Each active element activates its non-refractory cluster of neighboring elements immediately at each iteration interval, thus the conduction velocity through the matrix is determined by the cluster size and the iteration interval. That interval was chosen arbitrarily to be $4 \mathrm{~ms}$ to provide a reentry cycle length through the scar gap of $\sim 300 \mathrm{~ms}$. The conduction velocity within the scar gap is identical to that of the surrounding. After being excited, each element remains refractory to subsequent activation for an interval of $100 \mathrm{~ms}$, though this interval is arbitrary and was chosen to facilitate visualization of a sequence of activation wavefronts. The refractory time within the scar conduction gap is $260 \mathrm{~ms}$.

Programmed stimulation of the cardiac tissue plane is simulated by exciting specified locations within the tissue plane with a typical pacing protocol. In order to assess the range of VT inducibility, stimulation was tested at sequential points around the entire circumference of the scar while maintaining a constant distance from the outer edge of the scar. In these simulations, the last impulse of a $300 \mathrm{~ms}$ drive train is followed by a $220 \mathrm{~ms}$ extrastimulus. The simulation continues until no tissue is in the excited state or until a stable re-entrant circuit is demonstrated.

Case Description

\section{Case 1:}


A 57 year-old man without known cardiac disease presented with a wide complex tachycardia (WCT) at a rate of $270 \mathrm{bpm}$ requiring external cardioversion. Cardiac magnetic resonance imaging (MRI) revealed a mildly dilated LV and low-normal LV systolic function. Further evaluation showed no evidence of myocardial ischemia or arrhythmia on exercise stress testing.

At EPS, no arrhythmia was inducible despite burst and extra stimulus pacing from the RA, RVA, and RVOT. PES included up to 3 extrastimuli to a minimum coupling interval of $180 \mathrm{~ms}$ following two drive cycle lengths with and without isoproterenol (up to $12 \mathrm{mcg} / \mathrm{min}$ eliciting sinus tachycardia of $140 \mathrm{bpm}$ ). An RV voltage map identified a very small area of low bipolar voltage $(<1.5 \mathrm{mv})$ at the basal inferolateral RV raising concern for arrhythmogenic RV cardiomyopathy. Pericardial access was obtained and epicardial mapping revealed an extensive area of low voltage $(<1.5 \mathrm{mV}$, Figure 1$)$. PES was then performed from the RV epicardium with 3 extrastimuli following a drive train of $350 \mathrm{~ms}$ which reproducibly induced sustained monomorphic VT (cycle length 235ms) that was terminated with burst pacing. Following combined endocardial and epicardial ablation targeting the low voltage substrate, VT was no longer inducible with up to 3 extrasimuli from either the RV endocardium or epicardium.

\section{Case 2:}

A 79 year-old man with ischemic cardiomyopathy, cardiac resynchronization therapy ICD, and coronary artery bypass graft (CABG) surgery, presented with recurrent VT despite antiarrhythmic drug therapy, three prior endocardial ablation procedures, investigational irrigated intramural needle ablation, and bilateral cervical sympathectomy. ${ }^{11}$ In preparation for planned surgical epicardial ablation, non-invasive programmed stimulation (NIPS) was performed using his ICD to further characterize the ventricular arrhythmia. During light sedation, NIPS from the RV apical septal lead site with up to 3 extrastimuli down to ventricular refractoriness following drive cycle lengths of 400 and $600 \mathrm{~ms}$ failed to induce VT (Figure 2 Panel A). PES was then performed from the lateral LV utilizing the coronary sinus lead, where a pacing drive train of 600 ms without extrastimuli induced sustained monomorphic VT that had a RBBB right axis configuration, consistent with an apical lateral exit from his apical infarct scar (Figure 2 Panel B). VT was terminated with burst pacing. He subsequently underwent surgical epicardial mapping and cryoablation of a large LV epicardial scar, which rendered the VT non-inducible.

\section{Case 3:}

A 63 year-old woman with apical hypertrophic cardiomyopathy (HCM) presented with nearly incessant VT at $115 \mathrm{bpm}$ (RBBB superior axis) that failed to respond to beta blockers, amiodarone and mexiletine. VT was controlled with lidocaine that was stopped six hours prior to the study and she arrived in the EP laboratory in sinus rhythm. PES from the RVa with up to 3 extra stimuli down to refractoriness following a drive cycle length of $600 \mathrm{~ms}$ failed to induce VT. Polymorphic VT was induced with 400ms drive cycle length and two extra stimuli requiring external defibrillation. A substrate modification approach was planned and access to the LV was obtained by transseptal puncture. Burst pacing from the rim of the LV apical aneurysm at a cycle lengths of $300 \mathrm{~ms}$ readily induced the previously observed VT (Figure 3, panel A). Entrainment mapping was consistent with an isthmus at the lateral border of the aneurysm where the VT was successfully terminated (Figure 3, panel B). VT was no longer inducible following endocardial and epicardial ablation targeting the isthmus region.

\section{Case 4:}

A 64 year old man with ischemic cardiomyopathy and recurrent VT despite amiodarone, mexiletine and two prior ablation procedures presented for repeat VT ablation. PES from the RV apex with up to three extrastimuli down to refractoriness or a minimum coupling interval of $180 \mathrm{~ms}$ at two drive cycle lengths (400 and $600 \mathrm{~ms}$ ) did not induce VT. A substrate approach was planned and LV mapping revealed a large low voltage $(<1.5 \mathrm{mV})$ inferior and lateral scar. PES from the septal border zone of the scar with up to two extrastimuli following a basic drive cycle length of $600 \mathrm{~ms}$ induced four different sustained monomorphic VTs with cycle lengths of $268 \mathrm{~ms}$ to $566 \mathrm{~ms}$ and QRS morphologies consistent with origin from the mid-lateral and mid-inferoseptal aspects of the infarct scar. Following ablation, VTs were no longer inducible with PES 
from either the RV or LV. NIPS two days after the procedure via the RV lead of the patient's defibrillator did not induce VT.

\section{Case 5:}

A 44 year old man with previously normal ventricular function and a high burden of symptomatic premature ventricular contractions (PVCs) from various RV sites despite multiple previous ablations presented for repeat ablation. He was found to have a small area of endocardial low-voltage $(<1.5 \mathrm{mV})$ at the anterior RV outflow region. PES from the RV apex and basal septum with up to 4 extrastimuli following a drive cycle length of $350 \mathrm{~ms}$ did not induce any ventricular arrhythmia in the baseline state or following isoproterenol and epinephrine infusions. However, PES from the inferior RV free wall readily induced sustained monomorphic VT. Subsequent cardiac MRI and FDG PET were consistent with sarcoidosis. The details of his clinical course have been previously reported. ${ }^{12}$

\section{Case 6:}

A 36 year old man with a history of repaired Tetralogy of Fallot and recurrent VT despite sotalol therapy and prior catheter ablation presented for repeat ablation. PES from the RV apex and RV outflow tract with up to 4 extra stimuli following drive cycle lengths as short as $300 \mathrm{~ms}$ with and without isoproterenol (up to $4 \mathrm{mcg} / \mathrm{min}$ that elicited sinus tachycardia of $\sim 130 \mathrm{bpm}$ ) failed to induce VT. Single atrial extrastimuli during isoproterenol infusion induced sustained clinical VT (Figure 4). Programmed stimulation was then performed from the basal inferior RV septum and reproducibly induced VT. Activation and entrainment mapping revealed a circuit utilizing the infundibular septum requiring ablation from the RV and aortic root for interruption, as previously reported. ${ }^{13}$

\section{Illustrative Computer Simulation}

Programmed stimulation with a single extrastimulus following a basic drive was simulated for sites around the anatomic reentry circuit shown in figure 5 and Video 1 . In this model, proximity to the entrance/exit regions of the channel determine whether reentry will occur in response to an extrastimulus. S1 wavefronts propagate through the channel, the S2 wavefront blocks and fails to enter the adjacent entrance to the channel, but propagates to the other side with a sufficient to allow entry into the channel and initiation of reentry. This is an oversimplification of the complex anatomic and electrophysiologic heterogeneity in VT-causing substrate, but illustrates the marked effect that pacing site can have on inducibility of a simple circuit.

\section{Discussion}

This case series illustrates the potential importance of the site of stimulation when attempting to induce VT. In some patients, non-inducibility may be due to the inability of paced wavefronts from remote sites to engage potential reentry circuits, despite the use of multiple extrastimuli and adrenergic agents. The concept of enhanced VT induction probability by the addition of more stimulation sites, including the LV has been described previously. ${ }^{5,6,14,15}$ PES from the RV endocardium and LV epicardium has previously been compared in patients following surgical treatment of VT. ${ }^{16}$ The authors concluded that stimulation from either site yielded comparable results. The study was done post operatively, however, and was geared towards evaluating whether post-operative residual VT was predictive of future VT recurrence.

All six patients we describe in our case series had clinical VTs that were not induced with PES from the common RV sites (Table 1). All subjects had scar related re-entrant VT that was successfully induced from different alternate sites which markedly affected their subsequent clinical management. In five of the six patients (cases 1-5), the clinical VT was induced when PES was performed at a site anatomically closer to the expected critical VT isthmus. This observation supports that the proximity of the pacing site to the reentry circuit as an important factor beyond the probabilistic nature of PES yield in some patients. The pacing site is likely an important determinant of whether an extrastimulus wavefront will both reach the VT exit site sufficiently early to block due to refractoriness, and then propagate into the entrance and through the critical isthmus with sufficient delay to allow recovery at the exit site to allow VT initiation. This is 
likely related to both the anatomic and electrophysiologic properties of the scar and surrounding tissue as well as the distance from the stimulation site to various points in the circuit. At a stimulation site remote to the scar, or one where propagation velocity of the intervening tissue between the site and the circuit is slow, the paced wavefront may not reach the isthmus within the critical time window required to initiate VT. Conversely, by moving the stimulation site closer to the circuit, the paced wavefront is more likely to reach the critical isthmus and exit site with the appropriate relative timing to initiate reentry (figures 5 and video 1). Although our series suggests that close proximity to the reentry circuit is an important factor, perhaps by allowing premature stimuli to reach the circuit with less delay propagating through surrounding tissue, the direction from which the stimulated wave front arrives at the circuit is also likely important and a remote site may be more effective than a close site in some cases.

The sixth case illustrates the importance of the electrophysiologic properties of the intervening tissue between the stimulation site and the circuit in contrast to simple anatomic proximity. This patient had VT from the basal RV septum induced with atrial stimulation but not with aggressive stimulation from the RV apex and RVOT. In this case, right bundle branch block was present so atrial pacing activated the ventricle over the left bundle during atrial pacing, and perhaps resembled LV stimulation. The importance of atrial stimulation in the induction of idiopathic fascicular VT, which utilizes parts of the conduction system, has been described previously. ${ }^{17}$ Here, we posit that the conduction system was not formally involved in the circuit but did allow the paced wavefront to quickly engage the ventricular tissue proximate to the circuit despite its origin in the atrium.

The potential value of PES from different sites, including the RV epicardium in patients with suspected Arrhythmogenic Right Ventricular Cardiomyopathy (ARVC) is well demonstrated in the first case. The separation of the epicardial and endocardial layers in ARVC with long conduction delays from epicardium to endocardium have been previously described, ${ }^{18}$ but, to our knowledge, this is the first reported case of epicardial-specific VT inducibility in a patient with suspected ARVC.

This case series illustrates that alternate sites for PES, perhaps that are in close anatomic or electrophysiologic proximity to potential reentry circuits, are a reasonable consideration when trying to induce a clinical arrhythmia in patients with previously documented VT or in assessing arrhythmia risk in some patients with concern for VT (as illustrated by case 5). Alternative sites might also be considered when it is desirable to induce a specific clinical VT morphology, when only others are inducible with initial PES; as in patients who have repeated recurrence of a clinical VT after substrate guided ablation. Further studies are needed to evaluate the optimal pacing sites for assessing the value of inducibility during/following ablation procedures.

\section{Limitations}

The intent of this series is to illustrate the potential importance of alternative stimulation sites when assessing VT inducibility. It is unclear how often VT is only inducible from sites other than the RVA or RVOT. This is a retrospective case series without randomization of alternate stimulation sites or control for other factors which are inevitably changing during the course of the ablation procedure including the sympathetic tone, filling conditions, level of sedation, and absorption of local anesthetic, that can affect VT inducibility. The programmed stimulation was done as the procedure dictated. Hence it is difficult to provide guidance as to a specific approach to the problem.

The use of some alternate pacing sites may increase procedural risk. For example, most epicardial pacing sites require pericardial access. LV endocardial stimulation requires retrograde aortic or transseptal access to the LV, with the risks that this entails. In most cases, it seems likely that the additional risk is not warranted.

Clinical Implications: The site of PES has a marked effect on inducibility of VT in some patients. In some patients, PES from the RV apex and outflow regions will fail to expose clinically relevant VTs. The frequency with which this occurs is not certain. Stimulation from alternative sites is a reasonable consideration in selected patients. Our observations suggest that this may be especially useful when performing ablation in patients whose VT is not inducible despite previously documented clinical VTs. A PES site anatomically 
closer to the suspected reentry circuit is reasonable. Further studies are warranted to clarify the utility and selection of alternate pacing sites.

\section{Bibliography}

1. Stevenson WG, Soejima K. Catheter ablation for ventricular tachycardia. Circulation . 2007;115:27502760 .

2. Ghanbari H, Baser K, Yokokawa M, et al . Noninducibility in postinfarction ventricular tachycardia as an end point for ventricular tachycardia ablation and its effects on outcomes: a meta-analysis. Circ Arrhythm Electrophysiol . 2014;7:677-683.

3. Frankel DS, Mountantonakis SE, Zado ES, et al . Noninvasive programmed ventricular stimulation early after ventricular tachycardia ablation to predict risk of late recurrence. J Am Coll Cardiol . 2012;59:15291535 .

4. Hummel JD, Strickberger SA, Daoud E, et al . Results and efficiency of programmed ventricular stimulation with four extrastimuli compared with one, two, and three extrastimuli. Circulation . 1994;90:2827-2832.

5. Martínez-Rubio A, Stachowitz A, Borggrefe M, et al . Comparison of the results of programmed ventricular stimulation from the right ventricular apex and outflow tract: a randomized, prospective study.Eur Heart $J$ . 1995;16:1234-1243.

6. Robertson JF, Cain ME, Horowitz LN, et al . Anatomic and electrophysiologic correlates of ventricular tachycardia requiring left ventricular stimulation. Am J Cardiol . 1981;48:263-268.

7. Soejima K, Suzuki M, Maisel WH, et al . Catheter ablation in patients with multiple and unstable ventricular tachycardias after myocardial infarction: short ablation lines guided by reentry circuit isthmuses and sinus rhythm mapping. Circulation . 2001;104:664-669.

8. Stevenson WG, Sager PT, Natterson PD, Saxon LA, Middlekauff HR, Wiener I. Relation of pace mapping QRS configuration and conduction delay to ventricular tachycardia reentry circuits in human infarct scars. J Am Coll Cardiol . 1995;26:481-488.

9. Stevenson WG, Khan H, Sager P, et al . Identification of reentry circuit sites during catheter mapping and radiofrequency ablation of ventricular tachycardia late after myocardial infarction. Circulation . 1993;88:16471670 .

10. Stevenson WG, Friedman PL, Kocovic D, Sager PT, Saxon LA, Pavri B. Radiofrequency catheter ablation of ventricular tachycardia after myocardial infarction. Circulation . 1998;98:308-314.

11. Stevenson WG, Tedrow UB, Reddy V, et al. Infusion needle radiofrequency ablation for treatment of refractory ventricular arrhythmias. J Am Coll Cardiol . 2019;73:1413-1425.

12. Nakamura T, Tanigawa S, Schaeffer B, et al . A 16-year odyssey of cardiac sarcoid masquerading as idiopathic premature ventricular contractions and then arrhythmogenic cardiomyopathy. HeartRhythm Case Rep . 2018;4:260-263.

13. Kapel GFL, Reichlin T, Wijnmaalen AP, et al . Left-sided ablation of ventricular tachycardia in adults with repaired tetralogy of Fallot: a case series. Circ Arrhythm Electrophysiol . 2014;7:889-897.

14. Herre JM, Mann DE, Luck JC, et al . Effect of increased current, multiple pacing sites and number of extrastimuli on induction of ventricular tachycardia. Am J Cardiol . 1986;57:102-107.

15. Morady F, Hess D, Scheinman MM. Electrophysiologic drug testing in patients with malignant ventricular arrhythmias: importance of stimulation at more than one ventricular site. Am J Cardiol . 1982;50:1055-1060.

16. Dailey SM, Neal Kay G, Epstein AE, McGiffin DC, Kirklin JK, Plumb VJ. Comparison of endocardial and epicardial programmed stimulation for the induction of ventricular tachycardia. J Am Coll Cardiol . 1989;13:1608-1612. 
17. Gopi A, Nair SG, Shelke A, et al . A stepwise approach to the induction of idiopathic fascicular ventricular tachycardia. J Interv Card Electrophysiol . 2015;44:17-22.

18. Haqqani HM, Tschabrunn CM, Betensky BP, et al . Layered activation of epicardial scar in arrhythmogenic right ventricular dysplasia: possible substrate for confined epicardial circuits. Circ Arrhythm Electrophysiol . 2012;5:796-803.

19. Kucera JP, Kléber AG, Rohr S. Slow conduction in cardiac tissue, II: effects of branching tissue geometry. Circ Res . 1998;83:795-805.

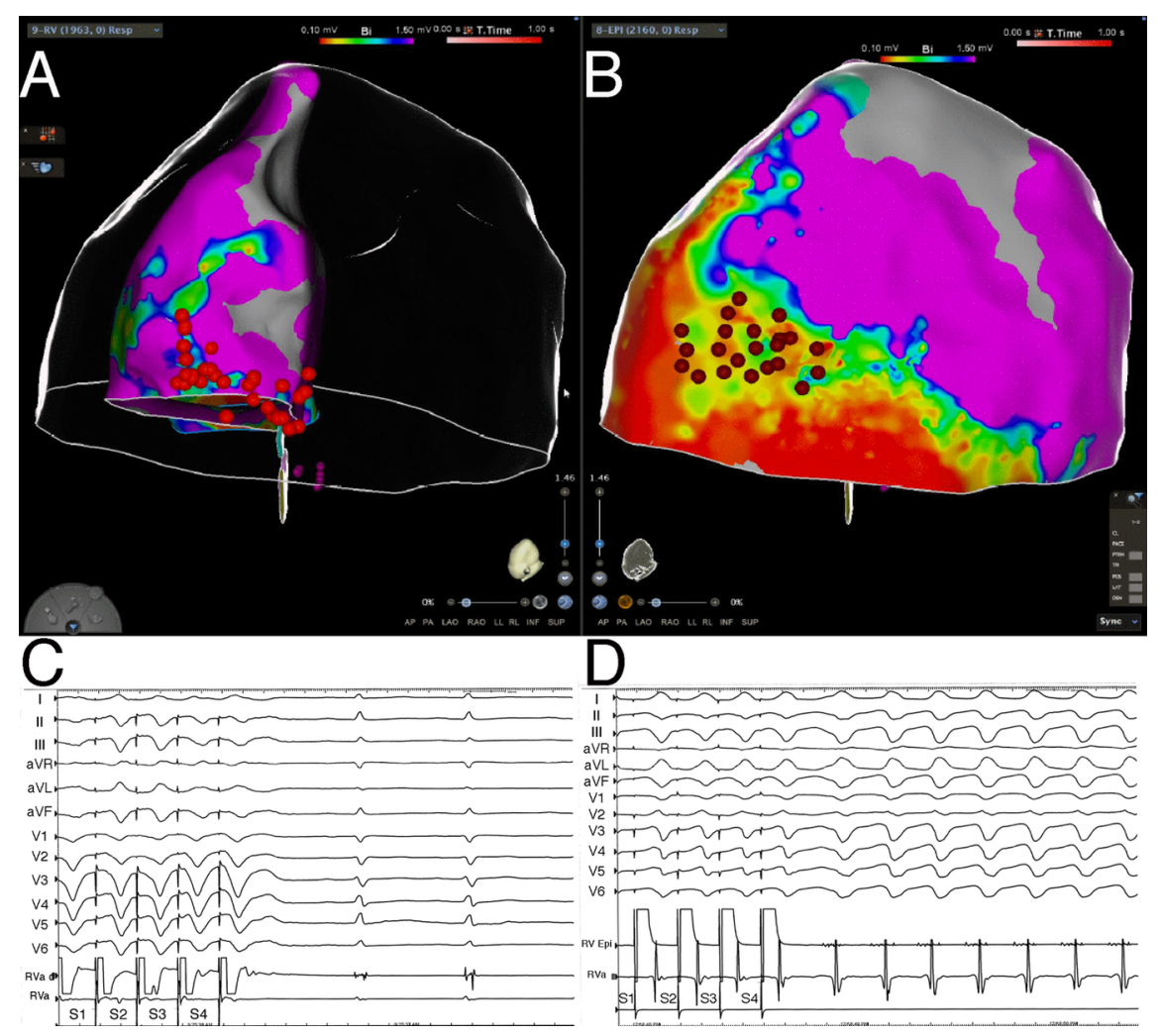

Figure 1: The discrepancy between the RV endocardial (Panel A) and epicardial (Panel B) voltage maps is illustrated in this patient with VT and suspected ARVC. Purple indicates bipolar voltage $>1.5 \mathrm{mV}$. While PES from the RVa (Panel C) did not induce VT, VT was readily induced with PES from the RV epicardium (Panel D). Maroon tags are ablation sites. 

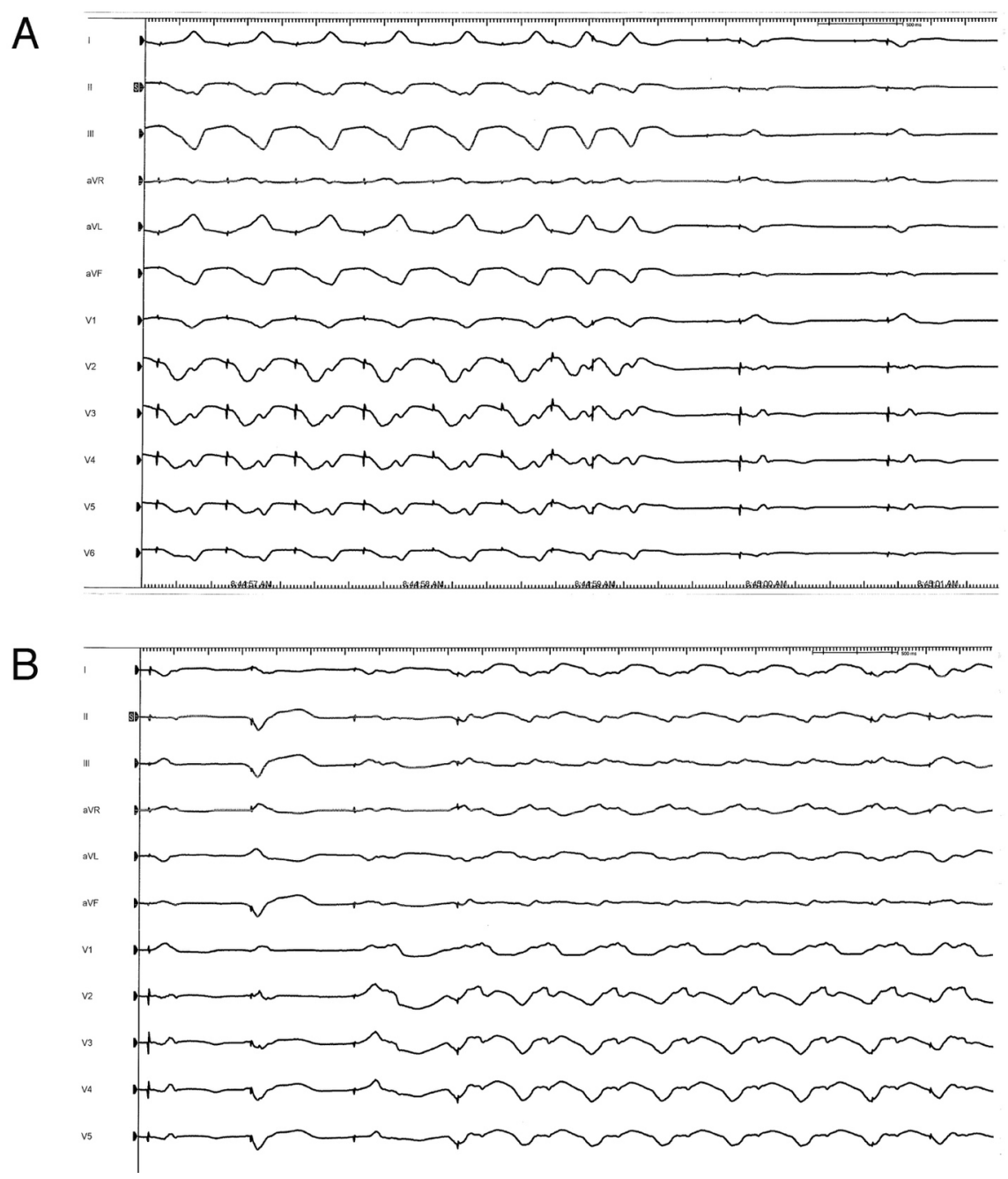

Figure 2. Non-invasive programmed stim from the RV ICD lead with up to 3 extrastimuli following a drive train of 400ms could not induce VT in this patient with ischemic cardiomyopathy and known history of VT (Panel A). PVCs were also present and pacing from the LV lead at $600 \mathrm{~ms}$ intermittently fusing with PVCs readily induced the clinical VT in the same patient. 

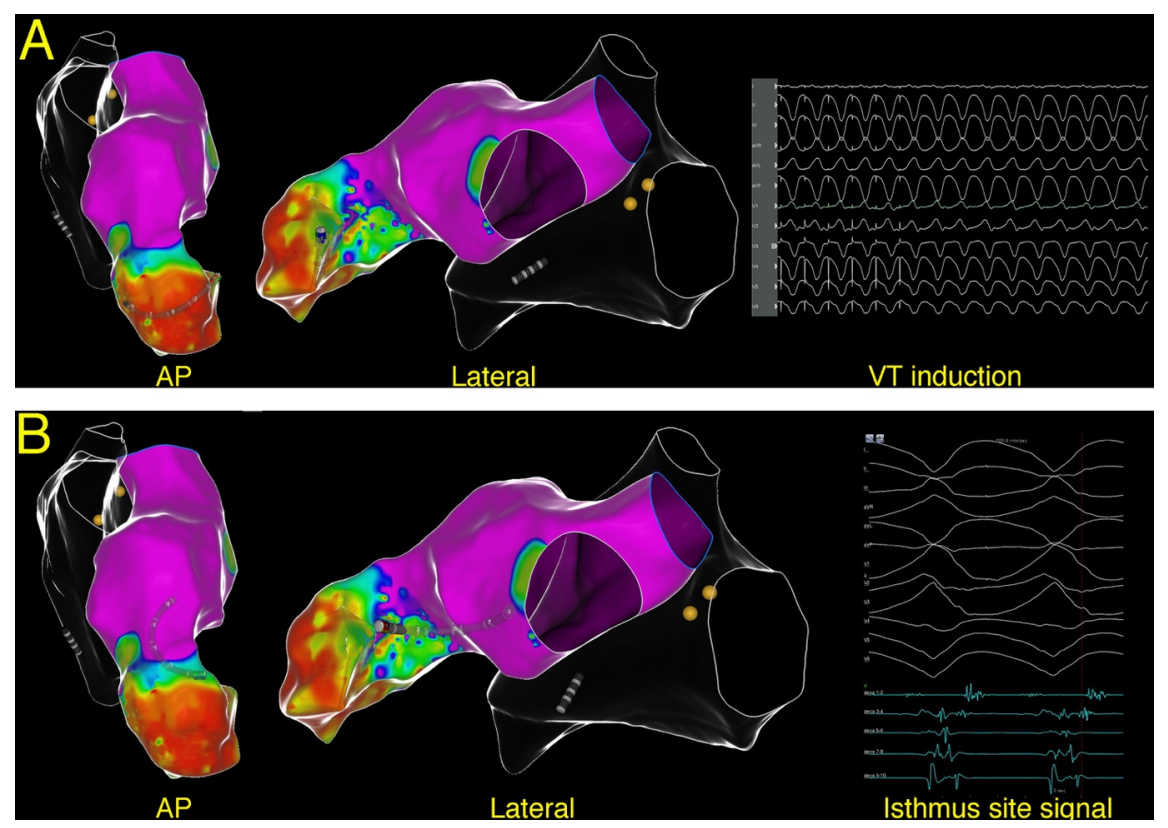

Figure 3. LV voltage maps (left panels) showing the pacing site location (distal electrodes of the mapping catheter) in a patient with hypertrophic cardiomyopathy and apical aneurysm are shown. A, the right panel shows the clinical VT was readily induced in this patient with burst pacing from the border of the LV apical aneurysm. The catheter position during stimulation is illustrated in the electroanatomic map on the left and middle of the panel. B, the right panel shows the local signal at the VT isthmus site where the clinical VT was successfully ablated. The left and middle of the panel show the catheter position at the isthmus site, which is in close proximity to the stimulation site that induced the clinical VT.
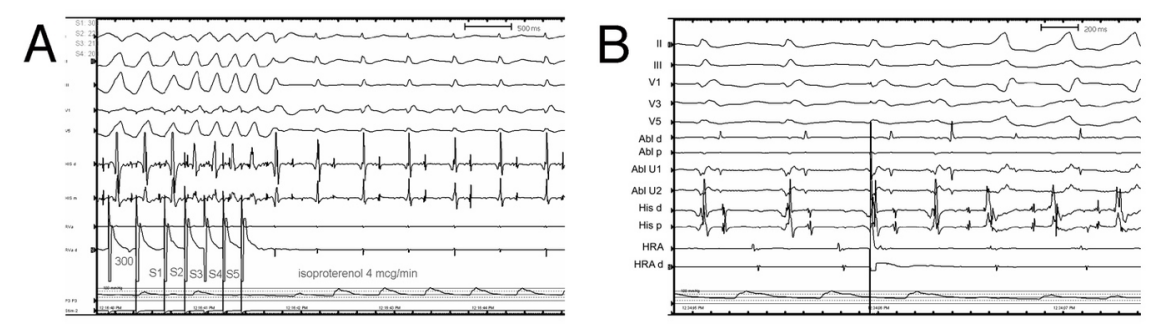

Figure 4: Panel A: programmed electrical stimulation did not induce VT from the RV apex despite using 4 extra stimuli with isoproterenol infusion in this patient with a history of repaired Tetralogy of Fallot and clinical VT. Panel B, a single atrial extrastimulus during isoproterenol infusion readily induced VT. 
A

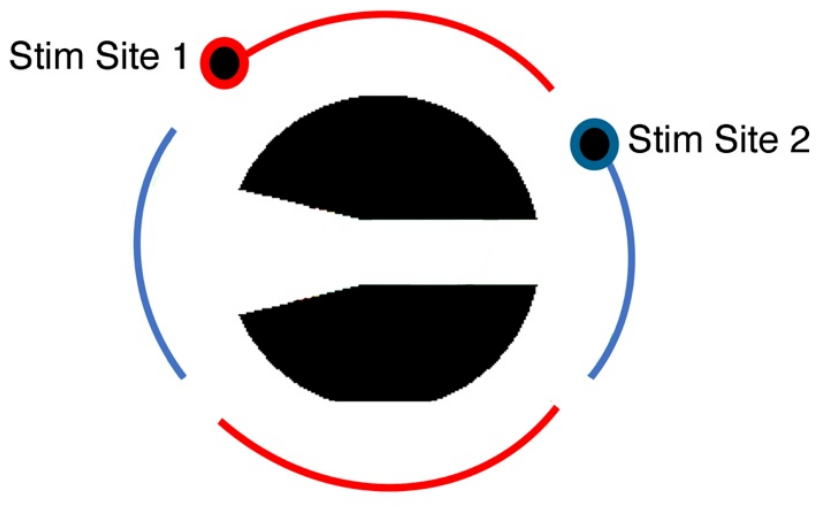

B

Stimulation Site 1

a

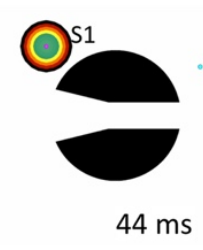

b

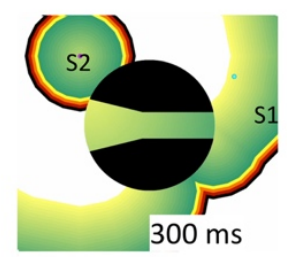

C
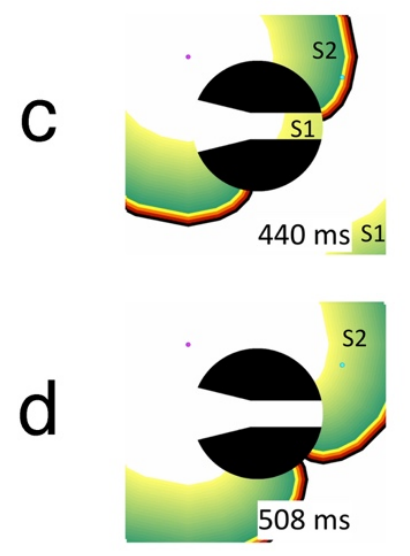

e

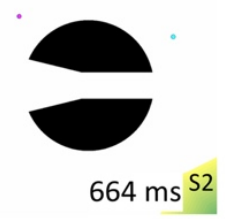

Stimulation Site 2

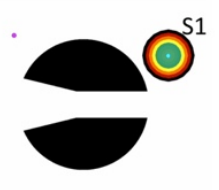

$44 \mathrm{~ms}$
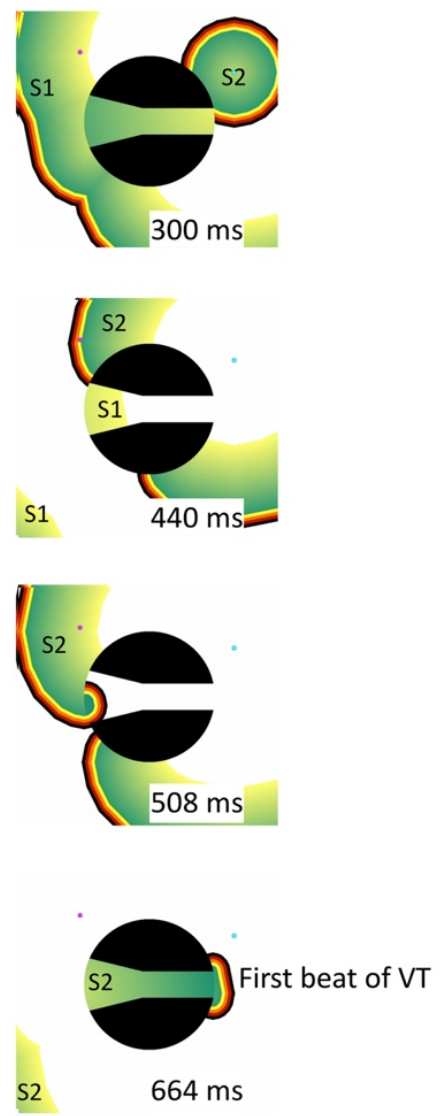
Figure 5: The relationship between the stimulation site and VT inducibility is illustrated using a computer model. In this model, unexcitable regions (black) create an asymmetrical channel, wider at the left-hand side, than at the right, creating anatomy that facilitates left to right conduction through the channel. ${ }^{19}$ Panel A. Simulations of the same stimulation sequence of stimuli were conducted to determine the range of sites over which re-entry was induced. Stimulation at sites along the blue line successfully induced VT, while stimulation from sites along the red line failed to do so. Panel B. Two separate simulations are shown with programmed stimulation applied at the 11 o'clock (Stimulation Site 1 where no VT was induced) and 2 o'clock positions (Stimulation Site 2 where VT was induced). The leading edge of the excitation wavefront is black and the trailing edge of refractoriness fades to green - yellow. The last beat of the drive train (S1) at $300 \mathrm{~ms}$ (panel a) and the onset and propagation of the extrastimulus beat (S2) at 220ms (panels b-e) are shown. With stimulation from Site 1 close to the broad end of the channel, the paced wavefronts encounter the ends of the channel with a timing that prevented reentry for all extrastimuli down to refractoriness. This is due to block of the S1 wavefront at the narrow end of the channel shortly before arrival of the S2 wavefront, such that the channel is refractory for the $\mathrm{S} 2$ wavefront. In contrast, Stimulation Site 2 is close to the narrow end of the channel. A premature stimulus encounters block at the narrow end, propagates to the entrance, then through the isthmus with sufficient time for the initial site of block to recover, and emerges from the channel producing the first reentrant beat.

Table 1: Clinical characteristics

\begin{tabular}{llllll}
\hline Case Number & Age & Gender & Type of CM & Ejection Fraction & AADs at the time of ablation \\
\hline 1 & 57 & M & ARVC & $56 \%$ (mild LV dilation) & None \\
2 & 79 & M & ICM & $20-25 \%$ & Mexiletine and Dofetilide (held 48h) \\
3 & 69 & F & HCM & $55-65 \%$ (apical aneurysm) & Amiodarone and Mexiletine (held 48h) \\
4 & 64 & M & ICM & $30-49 \%$ & Amiodarone and Mexiletine (held 48h) \\
5 & 44 & M & Sarcoid & normal & none \\
6 & 36 & M & TOF & normal & Sotalol (held) \\
\hline
\end{tabular}

ARVC: Arrhythmogenic Right Ventricular Cardiomyopathy, HCM: Hypertrophic Cardiomyopathy, ICD: Implantable cardioverter defibrillator, ICM: Ischemic Cardiomyopathy, LV: left ventricle, NICM: Non-ischemic Cardiomyopathy, RA: right atrium, RCC: the right coronary cusp of the aortic valve, RVa: Right ventricular apex, TOF: Tetralogy of Fallot, VT: Ventricular tachycardia. 